\title{
Chest wall perforator flaps for partial breast reconstruction: Surgical outcomes from a multicenter study
}

\author{
Soni Soumian ${ }^{1 *}$, Rishikesh Parmeshwar ${ }^{2 *}$, Mihir Chandarana ${ }^{3}$, Sekhar Marla ${ }^{1}$, \\ Sankaran Narayanan ${ }^{1}$, Geeta Shetty ${ }^{4}$ \\ ${ }^{1}$ Department of Breast and General Surgery, University Hospitals of North Midlands NHS Trust, Stoke-on-Trent; ${ }^{2}$ Department of Breast and \\ General Surgery, University Hospitals of Morecambe Bay NHS Foundation Trust, Lancaster; ${ }^{3}$ Department of General Surgery, Forth Valley \\ Royal Hospital NHS Trust, Larbert; ${ }^{4}$ Department of Breast and General Surgery, Sandwell and West Birmingham Hospitals NHS Trust, \\ Birmingham, UK
}

Background Perforator artery flaps based on the branches of intercostal arteries and lateral thoracic artery can be used for reconstruction after breast-conserving surgery (BCS). Although described more than a decade ago, these have not been adopted widely in clinical practice. We report on short-term and long-term surgical outcomes of partial breast reconstruction using chest wall perforator flaps from a prospective multicenter audit.

Methods All patients operated for BCS and partial breast reconstruction using intercostal artery perforator or lateral thoracic artery perforator flaps from January 2015 to October 2018 were included in the analysis. Oncoplastic breast surgeons with appropriate level of training performed all tumor excisions and reconstructions as a single-stage procedure. Patient characteristics, treatment details and surgical outcomes were noted. Specific outcomes recorded were margin re-excision and complication rates.

Results One hundred and twelve patients underwent the procedure in the given study period. The median age was 54 years. Median specimen weight was $62.5 \mathrm{~g}$ and median volume of excision was $121.4 \mathrm{~mL}$. Fifteen patients (13.39\%) underwent a margin re-excision for close or positive margins without additional morbidity. One patient required a completion mastectomy. Eight patients (7.14\%) had an early complication. None of the patients required a contralateral symmetrization procedure. The results were comparable across the participating centers.

Conclusions Chest wall artery perforator-based flaps are an excellent option for lateral and inferior quadrant partial breast reconstructions. The short and long-term surgical outcomes are comparable across sites and can be performed with minimal morbidity. Patient-reported outcome measures need to be studied.

Keywords Breast neoplasms / Perforator flap / Surgical flaps
Correspondence: Geeta Shetty Department of Breast and General Surgery, Birmingham City Hospital, Sandwell and West Birmingham Hospitals NHS Trust, Dudley road, Birmingham B18 70H, UK Tel: $+44-1215076180$ Fax: +44-1215075961 E-mail: g.shetty@nhs.net

\section{INTRODUCTION}

Oncoplastic breast surgery has resulted in better aesthetic out- comes and quality of life as compared to conventional breast surgery [1]. About $60 \%$ of patients diagnosed with breast cancer in the United Kingdom undergo breast-conserving surgery 
(BCS) and a subset would require oncoplastic procedures to ensure optimal cosmesis [2]. Oncoplastic techniques for BCS can be broadly divided into volume displacement and replacement techniques [3]. In small to medium sized breasts (up to C or $\mathrm{D}$ cup size), volume replacement techniques would be indicated, especially in relatively larger tumors. These require replacement of tissue into the tumor excision defect from a regional or distant site. The latissimus dorsi (LD) flap has been the workhorse of breast reconstruction for many decades and has also been used for partial breast reconstructions as a mini LD flap. However, sacrifice of one of the largest muscle of the body does lead to morbidity and impaired functional outcomes [4-6]. This, along with a better understanding of regional distribution of intercostal artery perforators (ICAPs) in the chest wall has led to evolution of muscle sparing perforator flaps with an aim to reduce donor site morbidity and achieve good aesthetic outcomes $[7,8]$.

Hamdi et al. [9-11] first reported on anatomy, classification, surgical technique and outcomes of partial breast reconstruction based on ICAP flaps. These flaps are based on the cutaneous perforator branches of posterior and anterior intercostal arteries. These vessels form an arcade between the aorta posteriorly and the internal mammary vessels anteriorly. The arcade is divided into vertebral, costal and muscular segments giving the dorsal, lateral and anterior perforators respectively [9]. Although described more than a decade ago, these flaps have not been adopted widely for partial breast reconstruction. A lateral thoracic artery perforator (LTAP) flap has been recently described and can be used exclusively or in combination with a lateral ICAP (LICAP) flap to reconstruct laterally situated breast excision defects [12]. The anterior intercostal artery-based flaps can be used for partial breast reconstruction for lower central and lower inner quadrant defects. A distinct advantage of LICAP and LTAP flaps is sparing of the LD muscle and the territory of the thoracodorsal artery perforator (TDAP) flap for future potential use. Other potential advantages include avoiding a mastectomy and providing skin cover for reconstruction if needed. Although reported surgical and aesthetic outcomes have been encouraging, these are limited to few centers routinely performing these procedures [13-18]. In collaboration with three other centers, we set up a multicenter prospective database and report on surgical outcomes in patients who underwent $\mathrm{BCS}$ and partial breast reconstruction using ICAP flaps.

\section{METHODS}

A multicenter prospective database was set up to include all patients undergoing for BCS and partial breast reconstruction with ICAP or LTAP flaps across four centers in the United Kingdom. This project was registered and approved by the respective local governance committees (registration numbers for each of the centers: CA12119, 1069, and 1668). All consecutive patients operated from January 2015 to October 2018 were included in the study. All patients deemed suitable for breast conservation and reconstruction with an ICAP or LTAP flap (up to $\mathrm{C}$ or D cup) were discussed in multidisciplinary team (MDT) meeting. The selection criterion was based on relative tumor to breast ratio for outer quadrants (excision volume of more than $20 \%$ ) and any excision in the lower inner and outer quadrants. For descriptive purposes, the area of the chest wall beneath the inframammary fold (IMF) was equally divided into three regions. A flap based on the medial segment was called the medial ICAP (MICAP) flap and that based on the middle third was the anterior ICAP (AICAP) flap. The MICAP flap was used for lower inner quadrant defects and the AICAP flap for lower central breast defects. For the outer third, LICAP flap was used. The LTAP and LICAP flaps were used for partial breast reconstruction after resection of tumors in the lateral quadrants of the breast-upper outer, outer central or lower outer. The LTAP was usually used in combination with LICAP flap for reconstruction. Thus, in a suitable patient, the choice of type of perforator flap used was primarily based on tumor location in the breast.

Patients are examined in the standing or sitting position for assessment of tumor location, post BCS defect and flap marking. For LICAP/LTAP flaps, the IMF, lateral mammary fold and posterior axillary fold are marked. The perforator vessels are typically located about $1-2 \mathrm{~cm}$ posterior to the lateral mammary fold and about $2-3 \mathrm{~cm}$ anterior to the posterior axillary fold arising from 3 rd to 7 th intercostal spaces. The LICAP perforators are usually found in the 4th to 6th intercostal spaces. The LTAP vessels can be traced vertically along the mid-axillary line for about $2-3 \mathrm{~cm}$, in most cases arising from 3rd to 5 th intercostal spaces thus differentiating them from the LICAP perforators. The width of the flap is based on the estimated breast defect and the available donor skin facilitating adequate closure. The length of the flap can be variable and up to $30 \mathrm{~cm}$ of the flap can be harvested without vascular compromise and this would again depend up on the amount of tissue needed for the defect (Fig. 1). For MICAP flap, the IMF is marked and the medial perforators can be located about $2-3 \mathrm{~cm}$ lateral to the sternal edge, about $1-2 \mathrm{~cm}$ inferior to the IMF. The flap extends laterally with the base of the flap situated medially. Similarly, the AICAP is based on the perforators located at 6-o'clock position 1-2 cm inferior to the IMF. The flap extends on either sides of the marked perforator and the tissue at 6-o'clock forms the base (Fig. 1). 
Breast surgeons trained in oncoplastic breast surgery performed the wide local excision and reconstruction in a single stage along with axillary surgery as indicated. Patients were positioned in supine position with arm extended for axillary surgery. For exposure of the LTAP/LICAP donor site a small sand bag was placed beneath the ipsilateral paraspinal area to achieve a tilt and the arm board was raised to support the arm. No intraoperative position change was required. A single dose of an antibiotic was given at the start of the procedure. A single incision was used for the wide local excision and reconstruction in all cases irrespective of the type of perforator flap used. The axil- lary procedure was performed through the same incision if feasible, in cases of LICAP and LTAP flaps or through a separate incision. The flaps were de-epithelized prior to placing them in the resection cavity. LICAP/LTAP flaps could also be used as propeller flaps with the donor site skin used to replace skin excision over the tumor. No magnification was required during the surgery. Assessment of specimen volume was performed by measuring the length, width and height of the wide local excision specimen. Drains were placed at donor site at the discretion of the operating surgeon. A separate drain was placed in the axilla for patients undergoing axillary nodal clearance. The drains

Fig. 1. Flap markings for reconstruction

For lateral intercostal artery

flap (A) and anterior intercostal artery-based flaps (B).
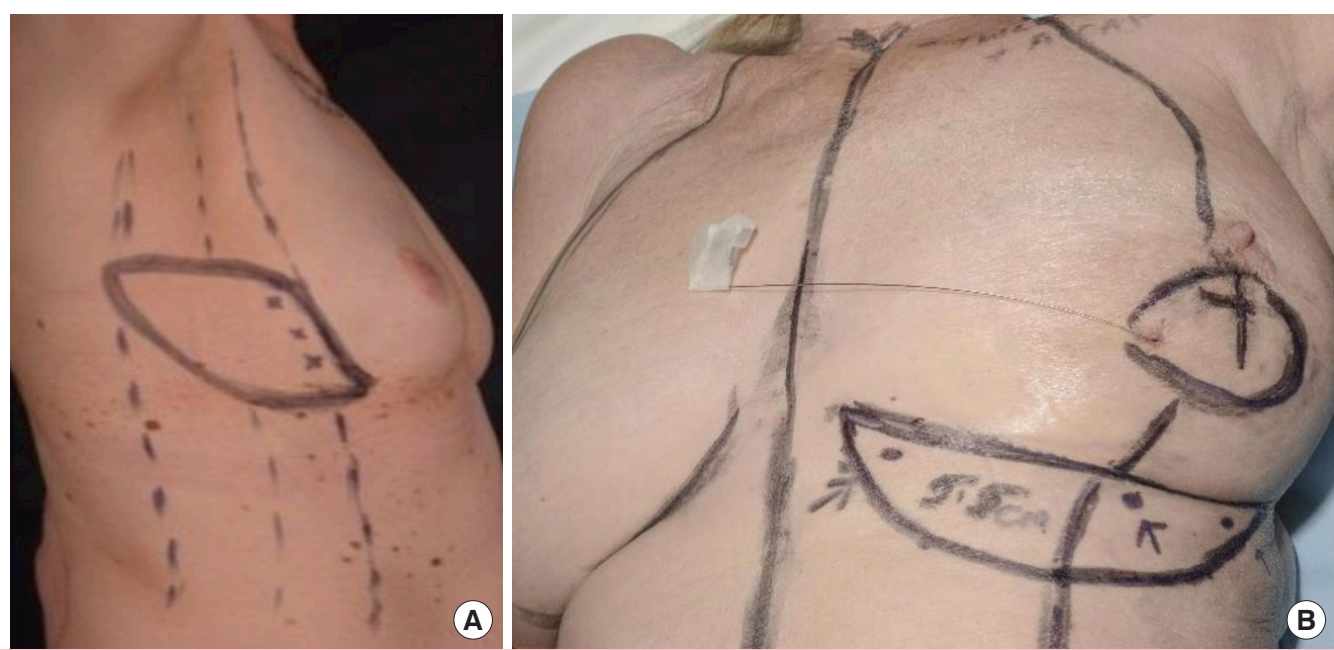

\section{Fig. 2. Surgical outcomes}

Preoperative $(A, B)$ and 3 months postoperative $(C, D)$ outcomes after a lateral intercostal artery perforator flap reconstruction.
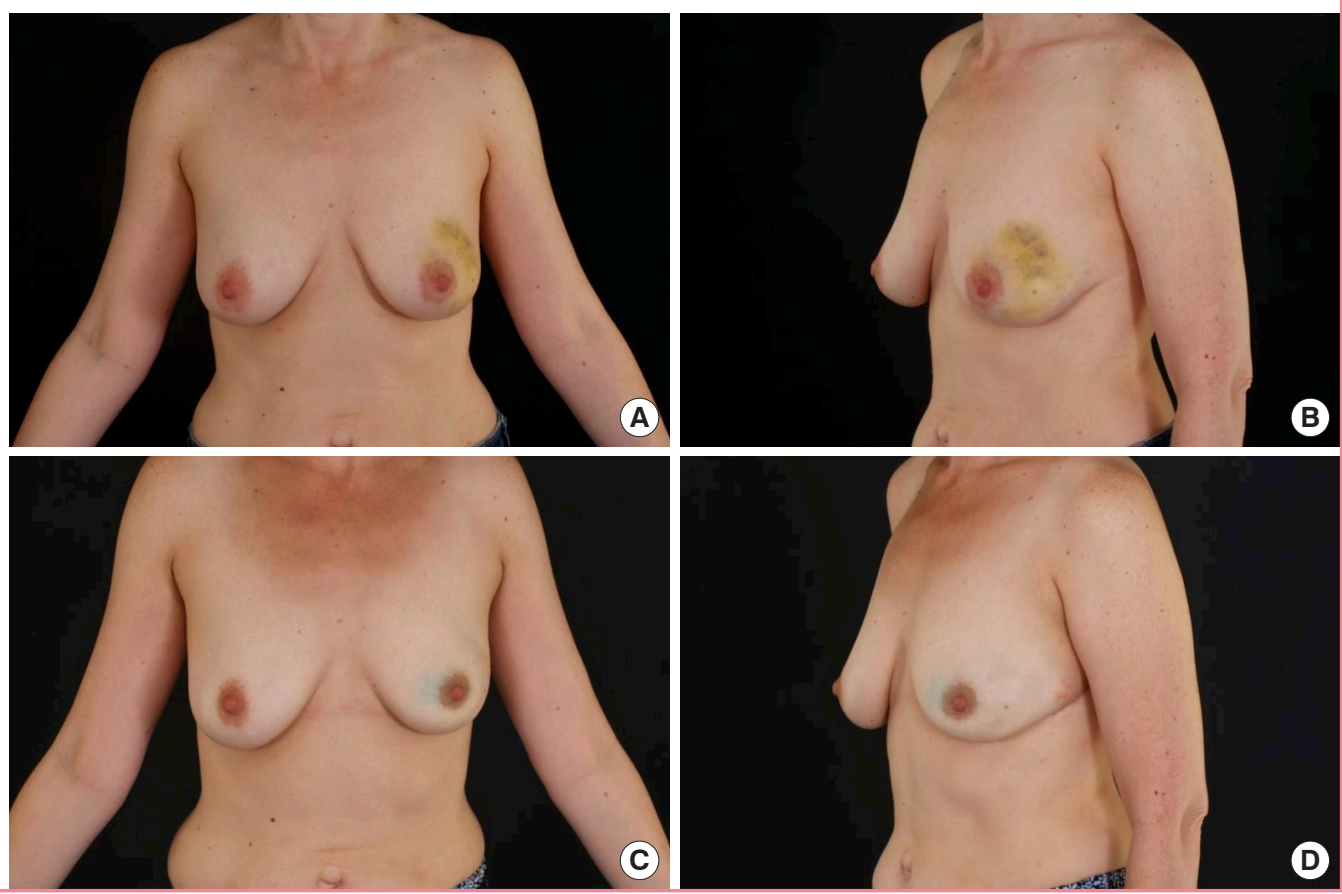
were managed postoperatively as per institutional protocol. $\mathrm{Pa}$ tients were discharged on the same day or next day as appropriate. Patients were reviewed in clinic after 1 week and 3 weeks of the primary surgery. Patient demographics, tumor characteristics and details of treatment received were recorded. The histological tumor size was used to record the T-stage. In patients who received neoadjuvant chemotherapy, this was the posttreatment histological residual tumor size. The resection volume was calculated by measuring the antero-posterior, medio-lateral and supero-inferior dimensions of the specimen. After 3 weeks, patients were followed-up routinely as per national guidelines. Specific outcomes recorded were margin re-excision rates, completion mastectomy rates, postoperative complications and aesthetic outcomes. All complications in the postoperative period were recorded. Complications occurring up to 90 days after the procedure were labeled as early complications and those after 90 days as delayed complications. All patients were referred for clinical photography before and after the procedure and after completion of adjuvant radiation therapy. Surgeons evaluated the aesthetic outcomes by comparison of preoperative and postoperative pictures and clinical examination (Fig. 2). Patients were discussed in the MDT meeting for postoperative outcomes and complications. The hospital ethical guidelines were followed in each center and patient consent was obtained to use their anonymized photographs for publication.

\section{RESULTS}

One hundred and twelve patients were operated in the given study period across four centers with a median follow-up period of 15 months. The demographic characteristics and treatment details are given in Table 1 . The median age of the cohort was 54 years and the median body mass index (BMI) was $26 \mathrm{~kg} / \mathrm{m}^{2}$. Twelve patients were smokers at the time of their surgery. The patients (85\%) were operated for invasive cancers and about $18 \%$ of the patients underwent an axillary nodal clearance along with the wide local excision. Perforator vessels could be localized using a hand-held Acoustic Doppler in all patients planned for the reconstruction. The LICAP and/or LTAP flaps were used in $78 \%$ of patients and the AICAP or MICAP flaps in $22 \%$ of patients. The median specimen weight was $62.5 \mathrm{~g}$ with a median volume of excision of $121.40 \mathrm{~mL}$. Most patients were discharged the same day or next day after surgery. Six patients had a hospital stay of 2 days and of these, four patients had a postoperative hematoma. The median histological tumor size was 25 $\mathrm{mm}$. Fifteen patients (13.39\%) had close or involved margins on histology and underwent re-excision of margins. These patients had their second surgery before commencing adjuvant
Table 1. Demographic characteristics and treatment details

\begin{tabular}{|c|c|}
\hline Characteristic & Value \\
\hline Total patients & 112 \\
\hline Age $(y r)$ & $54(27-83)$ \\
\hline BMI $\left(\mathrm{kg} / \mathrm{m}^{2}\right)$ & $26(19-37)$ \\
\hline \multicolumn{2}{|l|}{ ASA grade } \\
\hline ASA I & $60(53.57)$ \\
\hline ASA \| & $50(44.64)$ \\
\hline ASA III & $2(1.79)$ \\
\hline Current smokers & $12(10.71)$ \\
\hline \multicolumn{2}{|l|}{ Indication of surgery } \\
\hline Invasive tumor & $96(85.71)$ \\
\hline DCIS & $16(14.29)$ \\
\hline \multicolumn{2}{|l|}{ Tumor receptor status } \\
\hline ER+, HER2- & $77(68.75)$ \\
\hline ER+, HER2+ & $8(7.14)$ \\
\hline ER-, HER2+ & $6(5.36)$ \\
\hline ER-, HER2- & $5(4.46)$ \\
\hline Not applicable (DCIS) & $16(14.29)$ \\
\hline \multicolumn{2}{|l|}{ Tumor location } \\
\hline Upper outer & 65 (58.03) \\
\hline Outer central & $6(5.36)$ \\
\hline Lower outer & $14(12.50)$ \\
\hline Lower central & $12(10.71)$ \\
\hline Lower inner & $14(12.50)$ \\
\hline Multiple quadrants & $1(0.90)$ \\
\hline \multicolumn{2}{|l|}{ Management of axilla } \\
\hline Sentinel lymph node biopsy & $76(67.86)$ \\
\hline Axillary nodal clearance & $20(17.85)$ \\
\hline No axillary procedure & $16(14.29)$ \\
\hline \multicolumn{2}{|l|}{ Type of flap } \\
\hline LTAP & $3(2.68)$ \\
\hline LICAP with or without LTAP & $84(75.00)$ \\
\hline AICAP & $14(12.50)$ \\
\hline MICAP & $11(9.82)$ \\
\hline Specimen weight (g) & $62.50(21-231)$ \\
\hline Volume of excision (mL) & $121.40(36-412)$ \\
\hline \multicolumn{2}{|l|}{ Flap dimensions (cm) } \\
\hline Largest flap size (LICAP) & $15 \times 10$ \\
\hline Smallest flap size (LICAP) & $8 \times 3$ \\
\hline Average flap size & $12 \times 7$ \\
\hline Hospital stay (day) & $1(0-2)$ \\
\hline Histological tumor size (mm) & $25(0-110)$ \\
\hline$<2$ & $40(35.71)$ \\
\hline $2-5$ & $52(46.43)$ \\
\hline$>5$ & $11(9.82)$ \\
\hline Missing & $9(8.04)$ \\
\hline Neoadjuvant chemotherapy & $16(14.29)$ \\
\hline Adjuvant chemotherapy & 31 (27.68) \\
\hline Adjuvant radiation therapy & $110(98.21)$ \\
\hline Adjuvant endocrine therapy & 85 (75.89) \\
\hline Adjuvant trastuzumab & $13(11.61)$ \\
\hline Margin revision & $15(13.39)$ \\
\hline \multicolumn{2}{|c|}{$\begin{array}{l}\text { Values are presented as median (range) or number (\%). } \\
\text { BMI, body mass index; ASA, American Society of Anesthesiologists; DCIS, ductal } \\
\text { carcinoma in situ; ER, estrogen receptor; HER2, human epidermal growth factor } \\
\text { receptor 2; LTAP, lateral thoracic artery perforator; LICAP, lateral intercostal artery } \\
\text { perforator; AICAP, anterior intercostal artery perforator; MICAP, medial intercostal } \\
\text { artery perforator. }\end{array}$} \\
\hline
\end{tabular}


Fig. 3. Comparative outcomes across participating centers

Comparison of 24-hour discharge rates, margin re-excision rates and complication rates across the participating centers. ${ }^{\text {a) }}$ Center $\mathrm{C}$ includes patients from two centers as the same surgeon operated across both centers.

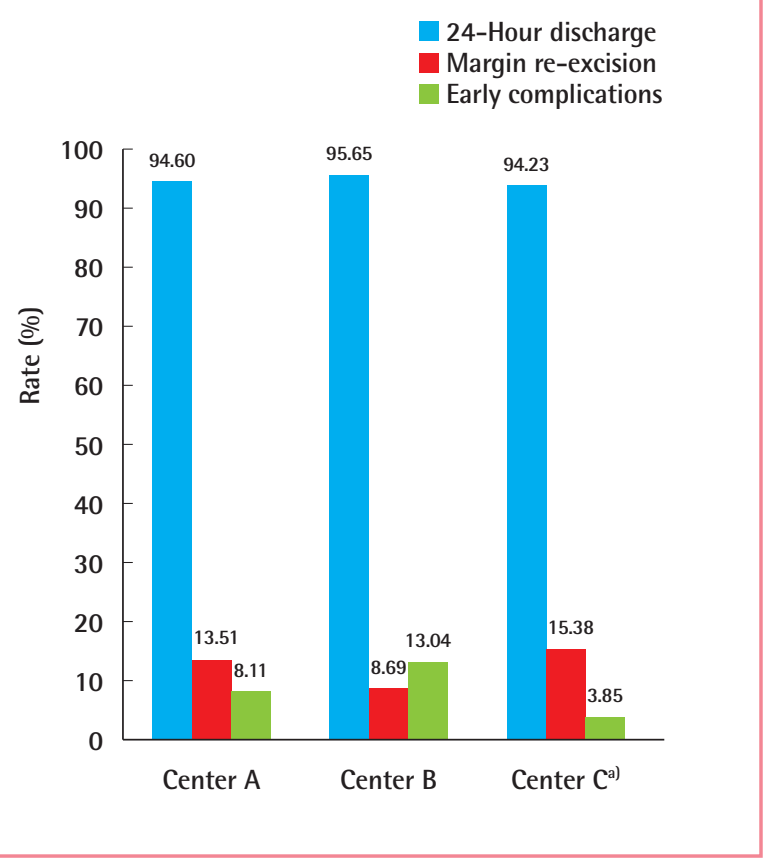

treatment and without any delay in adjuvant treatment. The primary surgeon performed the re-excision in all cases without any intraoperative concerns or postoperative complications secondary to flap handling. Five of the 15 patients who needed a margin revision had a primary tumor size of more than $50 \mathrm{~mm}$. One patient required a completion mastectomy due to persistent positive margin on re-excision. About $14 \%$ of patients received neoadjuvant chemotherapy and about $28 \%$ required adjuvant chemotherapy. One hundred and ten patients (98.21\%) received adjuvant radiation therapy.

Eight patients (7.14\%) had an early complication. Of these, four patients had a postoperative hematoma ( 2 axillary, 2 breast). Three patients were managed with surgical evacuation and one patient with a small breast hematoma was managed conservatively. One patient with LICAP flap reconstruction had a wound dehiscence treated with antibiotics and let to heal by secondary intention. One patient each had fat necrosis, surgical site pain and a breast seroma, all managed conservatively. No delayed complications were reported. None of the patients needed symmetrization procedure on the contralateral side. One patient had a systemic recurrence with multiple bony metastases and one patient had a local recurrence. Complication rates, margin re-excision rates and length of hospital stay were comparable across the participating centers (Fig. 3).

\section{DISCUSSION}

There have been various algorithms reported in literature to decide on the best mode of partial breast reconstruction based primarily on the volume of excision, breast size and ptosis [18-21]. Lateral and anterior chest wall perforator flaps are an excellent option for partial reconstruction of small to moderate breasts with limited ptosis for defects in lateral and inferior quadrants. Hamdi et al. [9] have reported extensively on ICAP flap reconstructions with their initial series including 20 patients. They further reported on 119 patients of partial breast reconstructions with a 4-year follow-up period. Of these, 93 patients had pedicled flap reconstructions, which included muscle-sparing LD, TDAP flaps, serratus anterior perforator flaps, superior epigastric flaps and ICAP flaps. All procedures were one-stage reconstructions [13]. McCulley et al. [12] introduced the use of LTAP perforator vessels for partial breast reconstruction and their series included 75 patients of LICAP and LTAP flaps used for immediate or delayed partial breast reconstruction. Roy and Tenovici [15] reported on using a 2-stage approach in patients with a high tumor to breast ratio. A wide local excision was performed initially with the resection cavity filled with saline. The LICAP/LTAP flap reconstruction was performed after the pathology results were available as a second surgery. Their study included 20 patients with a median follow-up of 23-month and a complication rate of $10 \%$. Carrasco-Lopez et al. [16] conducted a parallel cadaveric and clinical study on AICAP flaps and concluded that AICAP flap has a consistent vascularization with good perforators. The surgical and aesthetic outcomes in the above studies were reported to be satisfactory or excellent.

Our study was a prospective multicenter audit of ICAP or LTAP based partial breast reconstructions and to our knowledge, is the largest reported series till date. Twenty-five reconstructions $(22.3 \%)$ were based on the anterior ICAPs. Oncoplastic breast surgeons performed all operations as a single-stage procedure without contralateral symmetrization. No change in patient position was required and no magnification was used for the reconstruction. The tissue bearing the perforators was dissected and the perforator position was confirmed intraoperatively using a hand-held Acoustic Doppler without dissecting them and thus avoiding any chances of injury. Adequate reach of the flap was achieved in all cases. Eleven patients had a histological tumor size of more than $50 \mathrm{~mm}$, thus potentially avoiding a mastectomy or mammoplasty procedure in this set of patients. Five of these 11 patients (45.45\%) needed a margin revision, which could be performed without any additional morbidity. Only one patient required a completion mastectomy after a persistent positive margin on re-excision. Thus, we do not rou- 
tinely recommend a 2-stage approach even in larger tumors. Clinician reported subjective aesthetic outcomes based on clinical examination and comparison of preoperative and postoperative photographs were satisfactory or excellent in our study. Eight patients had a postoperative complication and of these, four were related to the flap reconstruction. There was one local recurrence in this series at a median follow-up of 15 months. A study by Ho et al. [22] has reported on oncological safety of oncoplastic breast surgery with tissue replacement techniques in 30 procedures with a follow-up of more than 4 years.

In our routine practice, ICAP flaps have become the procedure of choice for reconstruction in patients undergoing BCS for tumors in the lateral or lower breast quadrants with a high tumor to breast ratio. The lateral flaps can be either de-epithelized and turned over into the resection cavity where the breast skin overlying the tumor is preserved or used as propeller flaps to provide skin cover, based solely on the LTAP or single LICAP if skin excision is warranted as a part of the cancer resection. The incision of the LICAP/LTAP flaps is along the lateral mammary fold extending posteriorly to a variable extent and that for the AICAP/MICAP flap is along the IMF. The scars are well concealed postoperatively on frontal view images. They have a number of advantages as compared to LD flaps. There is no need for intraoperative position change or magnification for vessel dissection, unlikely need for contralateral symmetrization, LD and TDAP flaps can be spared for potential use in future and also reduces the morbidity secondary to sacrificing the LD muscle. The ICAP and LTAP flaps can be performed in smokers. The only possible contraindications include inadequate donor tissue or previous surgery in the donor area, which may indicate prior perforator damage. These procedures can be performed either by a breast surgeon trained in oncoplastic techniques or a combined team of breast and plastic surgeons.

Our study does have some drawbacks. It is a multicenter audit hence there could be some variation in patient selection and perioperative management among the surgeons. Follow-up period is relatively short, especially to comment on local recurrences. However, this was not the end-point of the study. A median follow-up period of 15 months should be satisfactory to comment on short-term and long-term surgical outcomes. It could be argued that a median tumor size of $25 \mathrm{~mm}$ is relatively small but the series includes post-neoadjuvant chemotherapy residual tumor sizes ( $14 \%$ in our study). Due to logistic issues, patient-reported outcome measures were not performed as a part of this study. Further follow-up studies including patientreported outcomes using a validated questionnaire have been planned. It was not possible to report on comparative outcomes between different volumes of excision or type of flaps due to small patient numbers in each subset.

\section{NOTES}

\section{Conflict of interest}

No potential conflict of interest relevant to this article was reported.

\section{Ethical approval}

The study was approved by the respective local governance committees (registration numbers for each of the centers: CA12119, 1069, and 1668) and performed in accordance with the principles of the Declaration of Helsinki. Written informed consents were obtained.

\section{Patient consent}

The patients provided written informed consent for the publication and the use of their images.

\section{Author contribution}

Conceptualization: Soumian S, Parmeshwar R, Chandarana M, Marla S, Narayanan S, Shetty G. Data Curation: Soumian S, Parmeshwar R, Chandarana M, Marla S, Narayanan S, Shetty G. Formal analysis: Soumian S, Parmeshwar R, Chandarana M, Marla S, Narayanan S, Shetty G. Methodology: Soumian S, Parmeshwar R, Chandarana M, Marla S, Narayanan S, Shetty G. Project administration: Soumian S, Parmeshwar R, Chandarana M, Marla S, Narayanan S, Shetty G. Visualization: Soumian S, Parmeshwar R, Chandarana M, Marla S, Narayanan S, Shetty G. Writing - original draft: Chandarana M, Soumian S. Writing - review and editing: Soumian S, Parmeshwar R, Chandarana M, Marla S, Narayanan S, Shetty G. Approval of the final draft: all authors.

\section{ORCID}

Soni Soumian https://orcid.org/0000-0002-7881-9695 Rishikesh Parmeshwar

$\begin{array}{ll} & \text { https://orcid.org/0000-0002-8162-2746 } \\ \text { Mihir Chandarana } & \text { https://orcid.org/0000-0002-9219-0184 } \\ \text { Sekhar Marla } & \text { https://orcid.org/0000-0002-2817-6468 } \\ \text { Sankaran Narayanan } & \text { https://orcid.org/0000-0002-1929-5887 } \\ \text { Geeta Shetty } & \text { https://orcid.org/0000-0002-7421-8139 }\end{array}$

\section{REFERENCES}

1. Chen JY, Huang YJ, Zhang LL, et al. Comparison of oncoplastic breast-conserving surgery and breast-conserving surgery alone: a meta-analysis. J Breast Cancer 2018;21:321-9. 
2. Jeevan R, Cromwell DA, Trivella M, et al. Reoperation rates after breast conserving surgery for breast cancer among women in England: retrospective study of hospital episode statistics. BMJ 2012;345:e4505.

3. Clough KB, Kaufman GJ, Nos C, et al. Improving breast cancer surgery: a classification and quadrant per quadrant atlas for oncoplastic surgery. Ann Surg Oncol 2010;17:1375-91.

4. Adams WP Jr, Lipschitz AH, Ansari M, et al. Functional donor site morbidity following latissimus dorsi muscle flap transfer. Ann Plast Surg 2004;53:6-11.

5. Lee KT, Mun GH. A systematic review of functional donorsite morbidity after latissimus dorsi muscle transfer. Plast Reconstr Surg 2014;134:303-14.

6. Smith SL. Functional morbidity following latissimus dorsi flap breast reconstruction. J Adv Pract Oncol 2014;5:181-7.

7. Taylor GI. The angiosomes of the body and their supply to perforator flaps. Clin Plast Surg 2003;30:331-42.

8. Saint-Cyr M, Wong C, Schaverien M, et al. The perforasome theory: vascular anatomy and clinical implications. Plast Reconstr Surg 2009;124:1529-44.

9. Hamdi M, Van Landuyt K, de Frene B, et al. The versatility of the inter-costal artery perforator (ICAP) flaps. J Plast Reconstr Aesthet Surg 2006;59:644-52.

10. Hamdi M, De Frene B. Pedicled perforator flaps in breast reconstruction. Semin Plast Surg 2006;20:73-8.

11. Hamdi M, Spano A, Van Landuyt K, et al. The lateral intercostal artery perforators: anatomical study and clinical application in breast surgery. Plast Reconstr Surg 2008;121: 389-96.

12. McCulley SJ, Schaverien MV, Tan VK, et al. Lateral thoracic artery perforator (LTAP) flap in partial breast reconstruction. J Plast Reconstr Aesthet Surg 2015;68:686-91.

13. Hamdi M. Oncoplastic and reconstructive surgery of the breast. Breast 2013;22 Suppl 2:S100-5.

14. Roy PG. One-stage vs. two-stage approach for partial breast reconstruction with lateral chest wall perforator flaps. Cancer Treat Res Commun 2016;9:56-61.

15. Roy PG, Tenovici AA. Staged approach to partial breast reconstruction to avoid mastectomy in women with breast cancer. Gland Surg 2017;6:336-42.

16. Carrasco-Lopez C, Julian Ibanez JF, Vila J, et al. Anterior intercostal artery perforator flap in immediate breast reconstruction: anatomical study and clinical application. Microsurgery 2017;37:603-10.

17. Kim JB, Kim DK, Lee JW, et al. The usefulness of pedicled perforator flap in partial breast reconstruction after breast conserving surgery in Korean women. Arch Plast Surg 2018; 45:29-36.

18. Yang JD, Kim MC, Lee JW, et al. Usefulness of oncoplastic volume replacement techniques after breast conserving surgery in small to moderate-sized breasts. Arch Plast Surg 2012;39:489-96.

19. Macmillan RD, McCulley SJ. Oncoplastic breast surgery: what, when and for whom? Curr Breast Cancer Rep 2016; 8:112-7.

20. Munhoz AM, Montag E, Gemperli R. Oncoplastic breast surgery: indications, techniques and perspectives. Gland Surg 2013;2:143-57.

21. Levine JL, Soueid NE, Allen RJ. Algorithm for autologous breast reconstruction for partial mastectomy defects. Plast Reconstr Surg 2005; 116:762-7.

22. Ho W, Stallard S, Doughty J, et al. Oncological outcomes and complications after volume replacement oncoplastic breast conservations: the Glasgow experience. Breast Cancer (Auckl) 2016;10:223-8. 